\title{
Polio Eradication in India: Need for Caution
}

Sir,

Global Polio Eradication Initiative (GPEI) is slowly but surely moving toward its culmination ${ }^{1}$. However, to achieve total success, it needs to tread extremely cautiously especially while dealing with polio situation in India. Currently, WHO has adopted an approach of sequentially targeting wild virus type- 1 and type-3, which is not only unethical but also fraught with certain risks. This is to be noted that routine immunization (RI) rates are abysmally low in the key states of UP and Bihar (12-23 months fully immunized rates $22.9 \%$, and $32.8 \%$, respectively (National family Health Surveys, Ministry of Health \& Family Welfare, Govt. of India, 2005-06.), and whatever immunity the children are possessing against all the 3 types of polioviruses is mainly achieved through supplementary immunization (SIA) rounds with tOPV, which have become a rarity since introduction of mOPV-1 in April 2005. The WHO decision to deliberately let loose the type 3 has resulted in huge outbreaks of type 3 in UP and Bihar in 2007. In recently conducted seroprevalence surveys of children aged 6-24 months of age in Moradabad, western UP revealed considerable immunity gap (around 15\%) against type 1 and moderate to huge gaps against types 2 and 3 despite receiving on an average 6 doses of OPV (Indian Council of Medical Research 2007-08 unpublished data). In similar serosurveys conducted in other developing countries in recent times ${ }^{2,3}$, much higher seroconversion rates against all the three types of polioviruses were seen. While analyzing any data pertaining to these two most populous states of India, one should remember that $1 \%$ children almost amounts to 400,000 children in UP alone. Additionally, there is a real risk of having an outbreak of circulating vaccinederived polioviruses, particularly of type 2 in UP.

Though right now there are no cases of type 1 in western UP despite considerable immunity gap, the WHO should not be carried away by this situation as there is a tendency of outbreaks of type 1 every four year in UP since 1998. On the contrary, WHO should find ways to close this immunity gap by whatever means available at their disposal. Employing inactivated poliovirus vaccine (IPV) would be one such option. Many recent articles including recommendations of National Institute of Health, Bethesda, USA, meeting on polio eradication in September, 2007 have advocated introduction of IPV even before eradication in hotspot states of India 1,4,5. The higher economic burden of employing IPV can also be resolved by proper planning and judicious use ${ }^{5}$.

Halting the transmission of type 1 from India by the year end is going to be the most significant achievement of GPEI in recent times, if it happens. However, even a cursory glance on the epidemiology of type 1 and 3 in India in last 5 yrs will reveal that there is periodic resurgence of these types in form of outbreaks particularly in UP and Bihar. Hence, GPEI needs to tread with extreme caution and should employ a multipronged strategy to close immunity gaps in high-risk areas of UP and Bihar before claiming success a real and long lasting one in these areas.

Vipin M. Vashishtha, MD, FIAP, Convener, Polio Eradication Committee of IAP, Consultant Pediatrician, Mangla Hospital, Shakti Chowk Bijnor-246701 (UP), India, E-mail:vipin@iappec.com

[DOI-10.1007/s12098-009-0126-X]

\section{REFERENCES}

1 Butcher J. Polio eradication nears the end game. Lancet Neurol 2008; 7: 292-293

2 Polio eradication: surveys of routine immunization coverage and seroprevalence against polioviruses, Yogyakarta Province, Indonesia. Wkly Epidemiol Rec 2008;83 : 45-48.

3 Seroprevalence of poliovirus antibodies among children in a Dominican community_Puerto Rico, 2002. MMWR Morb Mortal Wkly Rep 2005;54:580-581.

4 Paul Y. OPV cannot eradicate polio from India: Do we need any further evidence? Vaccine 2008; 26: 2058-2061.

5 Khan MM. Economics of polio vaccination in the posteradication era: Should OPV-using countries adopt IPV? Vaccine 2008; 26:2034-2040. 